\title{
Integrable spin-1 model with magnetic impurity
}

\author{
Jian Wang, ${ }^{1,2}$ Yusong Cao, ${ }^{1,2}$ Yi Qiao $\odot,{ }^{1, *}$ Junpeng Cao, ${ }^{1,2,3,4, \dagger}$ and Wen-Li Yang ${ }^{4,5,6,7, \sharp}$ \\ ${ }^{1}$ Beijing National Laboratory for Condensed Matter Physics, Institute of Physics, Chinese Academy of Sciences, Beijing 100190, China \\ ${ }^{2}$ School of Physical Sciences, University of Chinese Academy of Sciences, Beijing 100049, China \\ ${ }^{3}$ Songshan Lake Materials Laboratory, Dongguan, Guangdong 523808, China \\ ${ }^{4}$ Peng Huanwu Center for Fundamental Theory, Xian 710127, China \\ ${ }^{5}$ Institute of Modern Physics, Northwest University, Xian 710127, China \\ ${ }^{6}$ Shaanxi Key Laboratory for Theoretical Physics Frontiers, Xian 710127, China \\ ${ }^{7}$ School of Physical Sciences, Northwest University, Xian 710127, China
}

(Received 8 December 2020; revised 8 March 2021; accepted 9 April 2021; published 28 May 2021)

\begin{abstract}
We propose an integrable spin- 1 chain with a magnetic impurity whose spin is $1 / 2$. The integrability of the model is based on an operator solution of the associated reflection equation. Due to the existence of the impurity, the SU(3) symmetry of the bulk is broken. By using the nested algebraic Bethe ansatz, we obtain the exact solution of the system. The eigenvalues, eigenstates, and Bethe ansatz equations are given explicitly. The method provided in this paper can be generalized to other high-rank quantum integrable systems with magnetic impurities where the spins of the bulk and of impurities are different.
\end{abstract}

DOI: 10.1103/PhysRevResearch.3.023157

\section{INTRODUCTION}

Low-dimensional quantum many-body problems play an important role in the study of condensed matter, theoretical physics, and statistical mechanics [1]. Due to the strong interactions, it is very hard to study this kind of system because the mean-field approximation and perturbation theory cannot be applied. In order to overcome this difficulty, some numerical methods such as the density matrix renormalization group and tensor network [2,3] have been developed. Then a benchmark is needed to check the validity of numerical simulations and the new physical mechanism. The first candidate is the exact solutions because they can provide believable results. Fortunately, there do exist some quantum many-body systems which can be solved exactly such as one-dimensional interacting particles with contact potential [4], the Hubbard model [5], the supersymmetric $t-J$ model [6], and the Heisenberg spin chain [7]. The coordinate Bethe ansatz [8,9], algebraic Bethe ansatz [10-13], and $T-Q$ relation [14] are the typical methods to calculate the exact solutions of interacting systems. Later, in order to study high-rank quantum integrable systems, the nested algebraic Bethe ansatz was proposed [15-17].

Generic integrable boundary conditions include periodic, antiperiodic, and open boundary conditions. Recently, exactly strongly correlated systems with boundary reflections have attracted attention, because they have extensive appli-

\footnotetext{
*qiaoyi_joy@foxmail.com

†junpengcao@iphy.ac.cn

${ }^{\ddagger}$ wlyang@nwu.edu.cn
}

Published by the American Physical Society under the terms of the Creative Commons Attribution 4.0 International license. Further distribution of this work must maintain attribution to the author(s) and the published article's title, journal citation, and DOI. cations in the theories of quantum magnetism, topological physics, stochastic processes in nonequilibrium statistics, and open AdS/CFT duality. Many interesting phenomena such as Kondo problems, spiral phases, novel magnetic ordered states, and zero modes induced by boundary fields or magnetic impurities have been found [18-32].

For systems with open boundary conditions, besides the Yang-Baxter equation satisfied by two-body scattering matrices, integrability of the systems requires that the boundary reflection matrices should satisfy the reflection equation or its dual one [33-35].

Ordinarily, the solution of the reflection equation is a constant matrix [36-39]. Interestingly, the reflection equation can have operator solutions whose matrix elements are the operators $[29,32,40]$. These operators can be used to characterize the intrinsic degrees of freedom of magnetic impurities such as spin. Based on the exact solutions, the boundary bound states [29,40] and novel screened phases [32] are found, where the spins of impurities and of bulk particles are the same.

In this paper, we obtain a new operator solution of the reflection equation. Based on it, we construct a new exactly solvable model, where the spin of particles in the bulk is set at 1 , while the spin of the boundary impurity is set at $1 / 2$. By using the nested algebraic Bethe ansatz, we obtain the exact solution of the system. The method of constructing a new integrable Hamiltonian given in this paper can be generalized to other strongly correlated electronic systems with magnetic impurities.

We should note that the spin-1 bilinear biquadratic Heisenberg chain is very important. Some famous physical pictures such as the Haldane conjecture, where the biquadratic coupling is absent, and symmetry-protected topological phases, where free spin-1/2 is living on the boundaries in the gapped regime, are found [41-43]. Another interesting finding is that at the AKLT point $[44,45]$, the ground state of the system is 
the valence bond solid state. Bulk-boundary correspondence [44,45], a topological nature [46], and massless edge modes carrying a topological quantum number [47] are also obtained in this model.

This paper is organized as follows. In Sec. II, we introduce the integrable model Hamiltonian and show the details of construction. In Sec. III, we investigate the exact solution of the model. The commutative relations, energy spectrum, eigenstates, and Bethe ansatz equations are given explicitly. Section IV is devoted to concluding remarks.

\section{THE MODEL AND ITS INTEGRABILITY}

The integrable impurity model studied in this paper is characterized by the Hamiltonian

$$
\begin{aligned}
H= & \sum_{j=1}^{N-1}\left[\vec{S}_{j} \cdot \vec{S}_{j+1}+\left(\vec{S}_{j} \cdot \vec{S}_{j+1}\right)^{2}\right]+\frac{\sqrt{2}}{4} \vec{S}_{1} \cdot \vec{\sigma}_{\tau} \\
& -\frac{\sqrt{2}}{4}\left[S_{1}^{z}\left(\vec{S}_{1} \cdot \vec{\sigma}_{\tau}\right)+\left(\vec{S}_{1} \cdot \vec{\sigma}_{\tau}\right) S_{1}^{z}\right] \\
& -\frac{1}{4} S_{1}^{z}-\frac{1}{4}\left(S_{1}^{z}\right)^{2}+\frac{1}{2} \sigma_{\tau}^{z}+\frac{1-\sqrt{2}}{4} S_{1}^{z} \sigma_{\tau}^{z} \\
& +\frac{-3+2 \sqrt{2}}{4}\left(S_{1}^{z}\right)^{2} \sigma_{\tau}^{z},
\end{aligned}
$$

where $S_{j}^{\alpha}(\alpha=x, y, z)$ is the spin-1 operator of the bulk and $\sigma_{\tau}^{\alpha}$ is the spin-1/2 operator of the impurity on one side. Obviously, the interactions among particles in the bulk have SU(3) symmetry. This symmetry is broken due to the couplings between the boundary spin and the impurity spin. Meanwhile, system (1) is a high-spin system, thus the high-order coupling terms should be included to ensure integrability.

The integrability of Hamiltonian (1) is related to the $R$ matrix

$$
R_{i, j}(u)=\frac{u}{u+1}+\frac{1}{u+1} P_{i, j},
$$

where $u$ is the spectral parameter, and $P_{i, j}$ is the permutation operator with the matrix elements $\left[P_{i, j}\right]_{a c}^{b d}=\delta_{a d} \delta_{b c}$. Throughout this paper, we use standard notation. Let $V$ denote a three-dimensional linear space and $V_{\tau}$ denote the twodimensional linear space which belongs to the impurity. For any matrix $A \in \operatorname{End}(V), A_{j}$ is an embedding operator in the tensor space $V \otimes V \otimes \ldots$, which acts as $A$ in the $j$ th space and as an identity in the other factor spaces. For a matrix $R \in \operatorname{End}(V \otimes V), R_{i, j}$ is an embedding operator defined in the same tensor space, which acts as an identity in the factor spaces except for the $i$ th and $j$ th ones.

The $R$ matrix, (2), has the following properties.

$$
\begin{aligned}
& \text { Initial condition: } R_{i, j}(0)=P_{i, j} . \\
& \text { Unitarity: } R_{i, j}(u) R_{j, i}(-u)=\mathrm{id} .
\end{aligned}
$$

Crossing unitarity: $\quad R_{i, j}^{t_{i}}(u) R_{j, i}^{t_{i}}(-u-3)=\varphi(u) \times \mathrm{id}$,

$$
\varphi(u)=\frac{u(u+3)}{(u+1)(u+2)} .
$$

Fusion condition: $\quad R_{i, j}(u)=(u+1) \mathcal{R}_{i, j}(u)$,

$$
\mathcal{R}_{i, j}(-1)=-1+P_{i, j}=-2 P_{i, j}^{(-)} .
$$

Here $t_{i}$ denotes the transposition in the $i$ th space, $P_{i, j}$ is the permutation operator, and $R_{j, i}=P_{i, j} R_{i, j} P_{i, j}$. The operator $P_{i, j}^{(-)}$is actually a three-dimensional projector operator given by $P_{i, j}^{(-)}=\left|\psi_{1}\right\rangle\left\langle\psi_{1}|+| \psi_{2}\right\rangle\left\langle\psi_{2}|+| \psi_{3}\right\rangle\left\langle\psi_{3}\right|$, and the corresponding basis vectors are $\left|\psi_{1}\right\rangle=\frac{1}{\sqrt{2}}(\mid-$ $1,0\rangle-|0,-1\rangle),\left|\psi_{2}\right\rangle=\frac{1}{\sqrt{2}}(|-1,1\rangle-|1,-1\rangle)$, and $\left|\psi_{3}\right\rangle=$ $\frac{1}{\sqrt{2}}(|0,1\rangle-|1,0\rangle)$, where $|1\rangle,|0\rangle$, and $|-1\rangle$ are the eigenstates of the spin-1 operator $S^{z}$ with eigenvalues 1,0 , and -1 , respectively. The $R$ matrix, (2), satisfies the Yang-Baxter equation

$$
R_{i, j}(u-v) R_{i, k}(u) R_{j, k}(v)=R_{j, k}(v) R_{i, k}(u) R_{i, j}(u-v) .
$$

For integrable systems with open boundaries, besides the $R$ matrix, we should also consider the reflection matrix $K^{-}(u)$ and its dual one $K^{+}(u)$. The reflection matrix $K^{-}(u)$ satisfies the reflection equation

$$
\begin{aligned}
& R_{1,2}(u-v) K_{1}^{-}(u) R_{2,1}(u+v) K_{2}^{-}(v) \\
& =K_{2}^{-}(v) R_{1,2}(u+v) K_{1}^{-}(u) R_{2,1}(u-v),
\end{aligned}
$$

while the dual one $K^{+}(u)$ satisfies the dual reflection equation

$$
\begin{aligned}
& R_{1,2}(v-u) K_{1}^{+}(u) R_{2,1}(-u-v-3) K_{2}^{+}(v) \\
& =K_{2}^{+}(v) R_{1,2}(-u-v-3) K_{1}^{+}(u) R_{2,1}(v-u) .
\end{aligned}
$$

The general solutions of the reflection equations, (8) and (9), have been obtained, where all the matrix elements are constant [48-50]. In this paper, we seek the operator solutions. After some struggle, we obtain

$$
K^{-}(u)=\left(\begin{array}{ccc}
1-u^{2} & 0 & 0 \\
0 & 1+u^{2}+u\left(1+\sigma_{\tau}^{z}\right) & 2 u \sigma_{\tau}^{-} \\
0 & 2 u \sigma_{\tau}^{+} & 1+u^{2}+u\left(1-\sigma_{\tau}^{z}\right)
\end{array}\right) \equiv\left(\begin{array}{ccc}
k_{11}^{-}(u) & 0 & 0 \\
0 & k_{22}^{-}(u) & k_{23}^{-}(u) \\
0 & k_{32}^{-}(u) & k_{33}^{-}(u)
\end{array}\right) \text {, }
$$

where $\sigma_{\tau}^{ \pm}=\frac{1}{2}\left(\sigma_{\tau}^{x} \pm i \sigma_{\tau}^{y}\right)$. We note that the reflection matrix $K^{-}(u)$ acts on the three-dimensional auxiliary space whose elements are the operators acting on the Hilbert space of the impurity. It is clear that $K^{-}(u)$ is a block matrix and the impurity survives in the two-dimensional subspace, which is denoted $V_{\tau}$. The operator solution $K^{+}(u)$ of the dual reflection equation, (9), can be obtained similarly. In fact, the operator form of the dual reflection matrix can also be achieved by the mapping of $K^{+}(u)=K^{-}\left(-u-\frac{3}{2}\right)$. 
For simplicity, we consider the case where there is only one impurity in the system, thus the dual reflection matrix $K^{+}(u)$ is chosen as the identity matrix

$$
K^{+}(u)=\left(\begin{array}{lll}
1 & 0 & 0 \\
0 & 1 & 0 \\
0 & 0 & 1
\end{array}\right) .
$$

The monodromy matrix $T_{0}(u)$ and the reflecting one $\hat{T}_{0}(u)$ are defined as

$$
\begin{aligned}
& T_{0}(u)=R_{0, N}(u) R_{0, N-1}(u) \ldots R_{0,1}(u), \\
& \hat{T}_{0}(u)=R_{1,0}(u) R_{2,0}(u) \ldots R_{N, 0}(u),
\end{aligned}
$$

where $V_{0}$ is the auxiliary space, $V_{1} \otimes V_{2} \otimes \ldots \otimes V_{N}$ is the physical or quantum space, and $N$ is the total number of sites. The monodromy matrices satisfy the Yang-Baxter relations

$$
\begin{aligned}
& R_{1,2}(u-v) T_{1}(u) T_{2}(v)=T_{2}(v) T_{1}(u) R_{1,2}(u-v), \\
& R_{1,2}(u-v) \hat{T}_{1}(u) \hat{T}_{2}(v)=\hat{T}_{2}(v) \hat{T}_{1}(u) R_{1,2}(u-v) .
\end{aligned}
$$

The double-row monodromy matrix is

$$
\begin{aligned}
\mathcal{T}_{0}(u) & =T_{0}(u) K_{0}^{-}(u) \hat{T}_{0}(u) \\
& =\left(\begin{array}{ccc}
\mathcal{A}(u) & \mathcal{B}_{1}(u) & \mathcal{B}_{2}(u) \\
\mathcal{C}_{1}(u) & \mathcal{D}_{11}(u) & \mathcal{D}_{12}(u) \\
\mathcal{C}_{2}(u) & \mathcal{D}_{21}(u) & \mathcal{D}_{22}(u)
\end{array}\right) .
\end{aligned}
$$

The double-row monodromy matrix satisfies the reflection equation

$$
\begin{aligned}
& R_{1,2}(u-v) \mathcal{T}_{1}(u) R_{1,2}(u+v) \mathcal{T}_{2}(v) \\
& \quad=\mathcal{T}_{2}(v) R_{1,2}(u+v) \mathcal{T}_{1}(u) R_{1,2}(u-v) .
\end{aligned}
$$

The transfer matrix is

$$
t(u)=t r_{0}\left[K_{0}^{+}(u) \mathcal{T}(u)\right]=\mathcal{A}(u)+\mathcal{D}_{11}(u)+\mathcal{D}_{22}(u) .
$$

From the Yang-Baxter equation, (7), and reflection equation, (8), as well as the dual one, (9), one can prove that transfer matrices with different spectral parameters commutate with each other, $[t(u), t(v)]=0$. Thus $t(u)$ serves as the generating functional of all the conserved quantities, which ensures the integrability of the system. The model Hamiltonian, (1), is constructed by

$$
H=\left.\frac{1}{6} \frac{d}{d u} t(u)\right|_{u=0}+2 N-\frac{11}{6} .
$$

\section{EXACT SOLUTION}

\section{A. Commutative relations}

Now we solve model (1) by the nested algebraic Bethe ansatz, which includes two critical steps. One is the commutative relations among the matrix elements of the double-row monodromy matrix and the other is the vacuum state.

From the reflection equation, (15), we obtain

$$
\begin{gathered}
\mathcal{B}_{i}(u) \mathcal{B}_{j}(v)=\frac{r(u-v)_{l k}^{i j}}{u-v+1} \mathcal{B}_{k}(v) \mathcal{B}_{l}(u), \\
\mathcal{A}(u) \mathcal{B}_{j}(v)=\frac{(u-v-1)(u+v)}{(u+v+1)(u-v)} \mathcal{B}_{j}(v) \mathcal{A}(u)
\end{gathered}
$$

$$
\begin{gathered}
-\frac{1}{u+v+1} \mathcal{B}_{i}(u) \tilde{\mathcal{D}}_{i j}(v) \\
+\frac{2 v}{(u-v)(2 v+1)} \mathcal{B}_{j}(u) \mathcal{A}(v), \\
\tilde{\mathcal{D}}_{i j}(u) \mathcal{B}_{k}(v)=\frac{r(u+v+1)_{e f}^{i d} r(u-v)_{k j}^{f g}}{(u+v+1)(u-v)} \mathcal{B}_{d}(v) \tilde{\mathcal{D}}_{e g}(u) \\
-\frac{r(2 u+1)_{e j}^{i d}}{(2 u+1)(u-v)} \mathcal{B}_{d}(u) \tilde{\mathcal{D}}_{e k}(v) \\
+\frac{2 v}{2 u+1} \frac{r(2 u+1)_{k j}^{i d}}{(2 v+1)(u+v+1)} \mathcal{B}_{d}(u) \mathcal{A}(v),
\end{gathered}
$$

where the repeated indices should be summed and

$$
\begin{gathered}
\tilde{\mathcal{D}}_{i j}(u)=\mathcal{D}_{i j}(u)-\delta_{i j} \frac{1}{2 u+1} \mathcal{A}(u), \\
r(u)=\left(\begin{array}{cccc}
u+1 & 0 & 0 & 0 \\
0 & u & 1 & 0 \\
0 & 1 & u & 0 \\
0 & 0 & 0 & u+1
\end{array}\right) .
\end{gathered}
$$

We note that $r(u)$ is the $R$ matrix of the spin-1/2 Heisenberg model. According to definition (21), the transfer matrix, (16), reads

$$
t(u)=\frac{2 u+3}{2 u+1} \mathcal{A}(u)+\tilde{\mathcal{D}}_{11}(u)+\tilde{\mathcal{D}}_{22}(u) .
$$

\section{B. Vacuum state}

The vacuum state of the system takes the form

$$
|\Phi\rangle=\prod_{j=1}^{N}|0\rangle_{j}|0\rangle_{\tau} \equiv|\Omega\rangle|0\rangle_{\tau},
$$

where $|0\rangle_{j}=(1,0,0)^{t_{j}}$ and $|0\rangle_{\tau}=(1,0)^{t_{\tau}}$ are the vectors in the Hilbert spaces of the $j$ th spin and of the impurity, respectively. Obviously, $|0\rangle_{j}$ is the eigenstate of the spin operator $S_{j}^{z}$ with eigenvalue 1 and $|0\rangle_{\tau}$ is the eigenstate of impurity spin $\sigma_{\tau}^{z}$ with eigenvalue 1 . Here we should note that the double-row monodromy matrix $\mathcal{T}_{0}(u)$ and the reflection matrix $K_{0}^{-}(u)$ are defined in the three-dimensional auxiliary space $|0\rangle_{0}$. Thus the effect induced by the boundary magnetic impurity cannot be embodied in this step.

Acting the double-row monodromy matrix, (14), to the above product state, we obtain

$$
\begin{aligned}
& \mathcal{A}(u)|\Phi\rangle=k_{11}^{-}(u)|\Phi\rangle, \\
& \mathcal{D}_{11}(u)|\Phi\rangle=\left[\frac{k_{11}^{-}(u)}{2 u+1}+\left(k_{22}^{-}(u)-\frac{k_{11}^{-}(u)}{2 u+1}\right) b_{0}(u)\right]|\Phi\rangle, \\
& \mathcal{D}_{22}(u)|\Phi\rangle=\left[\frac{k_{11}^{-}(u)}{2 u+1}+\left(k_{33}^{-}(u)-\frac{k_{11}^{-}(u)}{2 u+1}\right) b_{0}(u)\right]|\Phi\rangle, \\
& \mathcal{D}_{12}(u)|\Phi\rangle=k_{23}^{-}(u) b_{0}(u)|\Phi\rangle, \\
& \mathcal{D}_{21}(u)|\Phi\rangle=k_{32}^{-}(u) b_{0}(u)|\Phi\rangle, \\
& \mathcal{B}_{n}(u)|\Phi\rangle \neq 0, \quad \mathcal{C}_{n}|\Phi\rangle=0, \quad n=1,2,
\end{aligned}
$$


where

$$
b_{0}(u)=\left(\frac{u}{u+1}\right)^{2 N} .
$$

From Eq. (25), we see that the operators $\mathcal{A}(u)$ and $\left\{\mathcal{D}_{i i}(u)\right\}$ acting on the vacuum state give the eigenvalues. The operators $\left\{\mathcal{B}_{i}(u)\right\}$ acting on the vacuum state generate other states and thus can be regarded as the creation operators. The operators $\left\{\mathcal{C}_{i}(u)\right\}$ acting on the vacuum state give 0 and thus can be regarded as the annihilation operators. From Eqs. (21) and (25), we also know that

$$
\begin{aligned}
& \tilde{\mathcal{D}}_{11}(u)|\Phi\rangle=\left(k_{22}^{-}(u)-\frac{k_{11}^{-}(u)}{2 u+1}\right) b_{0}(u)|\Phi\rangle, \\
& \tilde{\mathcal{D}}_{22}(u)|\Phi\rangle=\left(k_{33}^{-}(u)-\frac{k_{11}^{-}(u)}{2 u+1}\right) b_{0}(u)|\Phi\rangle .
\end{aligned}
$$

\section{Eigenstates}

Assume that the eigenstate of the system is

$$
\left.\left|u_{1}, \ldots, u_{M}\right| F\right\rangle=\mathcal{B}_{a_{1}}\left(u_{1}\right) \mathcal{B}_{a_{2}}\left(u_{2}\right) \ldots \mathcal{B}_{a_{M}}\left(u_{M}\right)|\Phi\rangle F^{a_{1} \ldots a_{M}},
$$

where $\left\{u_{l} \mid l=1, \ldots, M\right\}$ are the Bethe roots, $M$ is the number of Bethe roots, and $F^{a_{1} \ldots a_{M}}$ are the related nested algebraic Bethe states including the impurity effect, which is determined in the next step. We expect that the transfer matrix $t(u)$ acting on the assumed eigenstate, (27), can give the eigenvalue. Because we only know the behaviors of the transfer matrix $t(u)$ acting on the vacuum state, (24), we should exchange the order between $\mathcal{B}_{a_{l}}\left(u_{l}\right)$ and $\mathcal{A}(u),\left\{\tilde{\mathcal{D}}_{n n}(u)\right\}$ until the transfer matrix $t(u)$ can act on the vacuum state, (24).

Repeatedly using the commutative relations (18)-(20) and considering properties (25) and (26), after some tedious calculations, we arrive at

$$
\begin{aligned}
\left.t(u)\left|u_{1}, \ldots, u_{M}\right| F\right\rangle= & \left.\left\{\frac{(2 u+3) k_{11}^{-}(u)}{2 u+1} \prod_{j=1}^{M} \frac{\left(u-u_{j}-1\right)\left(u+u_{j}\right)}{\left(u-u_{j}\right)\left(u+u_{j}+1\right)}+b_{0}(u) \prod_{j=1}^{M} \frac{1}{\left(u+u_{j}+1\right)\left(u-u_{j}\right)} t^{(1)}\left(u,\left\{u_{l}\right\}\right)\right\}\left|u_{1}, \ldots, u_{M}\right| F\right\rangle \\
& +\sum_{j=1}^{M} \Lambda_{j}(u) \mathcal{B}_{a_{1}}\left(u_{1}\right) \ldots \mathcal{B}_{a_{j-1}}\left(u_{j-1}\right) \mathcal{B}_{a_{j}}(u) \mathcal{B}_{a_{j+1}}\left(u_{j+1}\right) \ldots \mathcal{B}_{a_{M}}\left(u_{M}\right)|\Phi\rangle F^{a_{1} \ldots a_{M}} .
\end{aligned}
$$

Here $t^{(1)}\left(u,\left\{u_{l}\right\}\right)$ is the nested transfer matrix with the form

$$
t^{(1)}\left(u,\left\{u_{l}\right\}\right)=\operatorname{tr}_{0}\left[\bar{K}_{0}^{+}(u) r_{0,1}\left(u+u_{1}+1\right) \ldots r_{0, M}\left(u+u_{M}+1\right) \bar{K}_{0}^{-}(u) r_{M, 0}\left(u-u_{M}\right) \ldots r_{1,0}\left(u-u_{1}\right)\right],
$$

where $\bar{K}^{ \pm}(u)$ are the nested reflection matrices

$$
\bar{K}^{+}(u)=\left(\begin{array}{ll}
1 & 0 \\
0 & 1
\end{array}\right), \quad \bar{K}^{-}(u)=\left(\begin{array}{cc}
k_{22}^{-}(u)-\frac{k_{11}^{-}(u)}{2 u+1} & k_{23}^{-}(u) \\
k_{32}^{-}(u) & k_{33}^{-}(u)-\frac{k_{11}^{-}(u)}{2 u+1}
\end{array}\right),
$$

and $r_{0, l}(u)$ is the nested $R$ matrix given by (22). We diagonalize the nested transfer matrix $t^{(1)}\left(u,\left\{u_{l}\right\}\right)$ in the next subsection. Denote the eigenvalue of $t^{(1)}\left(u,\left\{u_{l}\right\}\right)$ as $\Lambda^{(1)}\left(u,\left\{u_{l}\right\}\right)$, which is given by Eq. (46) in the following. $\Lambda_{j}(u)$ is the unwanted term

$$
\begin{aligned}
\Lambda_{j}(u)= & \frac{2 u+3}{\left(u+u_{j}+1\right)\left(u-u_{j}\right)} \frac{1}{2 u_{j}+3}\left[\frac{2\left(2 u_{j}+3\right) u_{j}\left(1-u_{j}^{2}\right)}{2 u_{j}+1} \prod_{l \neq j}^{M} \frac{\left(u_{j}-u_{l}-1\right)\left(u_{j}+u_{l}\right)}{\left(u_{j}-u_{l}\right)\left(u_{j}+u_{l}+1\right)}\right. \\
& \left.-b_{0}\left(u_{j}\right) \prod_{l \neq j}^{M} \frac{1}{\left(u_{j}+u_{l}+1\right)\left(u_{j}-u_{l}\right)} \Lambda^{(1)}\left(u_{j},\left\{u_{l}\right\}\right)\right] .
\end{aligned}
$$

From Eq. (28), we see that if the assumed state, (27), is indeed the eigenstate of the transfer matrix $t(u)$, the unwanted terms in Eq. (28) should vanish, which gives the constraints of the Bethe roots $\left\{u_{l}\right\}$. Putting $\Lambda_{j}(u)=0$, we obtain the first set of Bethe ansatz equations

$$
\begin{aligned}
& \frac{\left(2 u_{j}+3\right)\left(1-u_{j}^{2}\right)}{2 u_{j}+1} \prod_{l=1}^{M}\left(u_{j}-u_{l}-1\right)\left(u_{j}+u_{l}\right)=-b_{0}\left(u_{j}\right) \Lambda^{(1)}\left(u_{j},\left\{u_{l}\right\}\right), \\
& j=1,2, \ldots, M,
\end{aligned}
$$

where $\Lambda^{(1)}\left(u_{j},\left\{u_{l}\right\}\right)$ is the value of $\Lambda^{(1)}\left(u,\left\{u_{l}\right\}\right)$ at the point $u_{j}$. The corresponding eigenvalues $\Lambda(u)$ of the transfer matrix $t(u)$ are

$$
\Lambda(u)=\frac{(2 u+3)\left(1-u^{2}\right)}{2 u+1} \prod_{j=1}^{M} \frac{\left(u-u_{j}-1\right)\left(u+u_{j}\right)}{\left(u-u_{j}\right)\left(u+u_{j}+1\right)}+b_{0}(u) \prod_{j=1}^{M} \frac{1}{\left(u+u_{j}+1\right)\left(u-u_{j}\right)} \Lambda^{(1)}\left(u,\left\{u_{l}\right\}\right),
$$

where the Bethe roots $\left\{u_{l}\right\}$ should satisfy the Bethe ansatz equations, (32). 


\section{Nested eigenvalue problem}

The rest of the task is to determine the value of $\Lambda^{(1)}\left(u,\left\{u_{l}\right\}\right)$. Define the nested single-row monodromy matrices $T_{0}^{(1)}(u)$ and $\hat{T}_{0}^{(1)}(u)$ as

$$
\begin{aligned}
& T_{0}^{(1)}(u)=r_{0,1}\left(u+u_{1}+1\right) \ldots r_{0, M}\left(u+u_{M}+1\right), \\
& \hat{T}_{0}^{(1)}(u)=r_{M, 0}\left(u-u_{M}\right) \ldots r_{1,0}\left(u-u_{1}\right) .
\end{aligned}
$$

The nested double-row monodromy matrix is

$$
\mathcal{T}_{0}^{(1)}(u)=T_{0}^{(1)}(u) \bar{K}_{0}^{-}(u) \hat{T}_{0}^{(1)}(u)=\left(\begin{array}{ll}
\mathcal{A}^{(1)}(u) & \mathcal{B}^{(1)}(u) \\
\mathcal{C}^{(1)}(u) & \mathcal{D}^{(1)}(u)
\end{array}\right)
$$

which satisfies the reflection equation

$$
\begin{aligned}
& r_{1,2}(u-v) \mathcal{T}_{1}^{(1)}(u) r_{2,1}(u+v+1) \mathcal{T}_{2}^{(1)}(v) \\
& \quad=\mathcal{T}_{2}^{(1)}(v) r_{1,2}(u+v+1) \mathcal{T}_{1}^{(1)}(u) r_{2,1}(u-v) .
\end{aligned}
$$

Then the nested transfer matrix, (29), reads

$$
t^{(1)}\left(u,\left\{u_{l}\right\}\right)=\operatorname{tr}_{0}\left[\bar{K}_{0}^{+}(u) \mathcal{T}_{0}^{(1)}(u)\right]=\mathcal{A}^{(1)}(u)+\mathcal{D}^{(1)}(u) .
$$

From the reflection equation, (35), we obtain the commutative relations among the elements of the nested double-row monodromy matrix

$$
\begin{aligned}
\mathcal{B}^{(1)}(u) \mathcal{B}^{(1)}(v)= & \mathcal{B}^{(1)}(v) \mathcal{B}^{(1)}(u), \\
\mathcal{A}^{(1)}(u) \mathcal{B}^{(1)}(v)= & \frac{(u+v+1)(u-v-1)}{(u-v)(u+v+2)} \mathcal{B}^{(1)}(v) \mathcal{A}^{(1)}(u)+\frac{u+v+1}{(u-v)(u+v+2)} \mathcal{B}^{(1)}(u) \mathcal{A}^{(1)}(v)-\frac{1}{u+v+2} \mathcal{B}^{(1)}(u) \mathcal{D}^{(1)}(v), \\
\mathcal{D}^{(1)}(u) \mathcal{B}^{(1)}(v)= & \frac{(u-v+1)(u+v+2)}{(u-v)(u+v+1)} \mathcal{B}^{(1)}(v) \mathcal{D}^{(1)}(u)+\frac{u-v+1}{(u-v)(u+v+1)} \mathcal{A}^{(1)}(v) \mathcal{B}^{(1)}(u) \\
& -\frac{u+v+2}{(u-v)(u+v+1)} \mathcal{B}^{(1)}(u) \mathcal{D}^{(1)}(v)-\frac{1}{(u-v)(u+v+1)} \mathcal{A}^{(1)}(u) \mathcal{B}^{(1)}(v) .
\end{aligned}
$$

Define

$$
\tilde{\mathcal{D}}^{(1)}(u)=\mathcal{D}^{(1)}(u)-\frac{1}{2 u+2} \mathcal{A}^{(1)}(u)
$$

then we have

$$
\begin{aligned}
\mathcal{A}^{(1)}(u) \mathcal{B}^{(1)}(v)= & \frac{(u+v+1)(u-v-1)}{(u-v)(u+v+2)} \mathcal{B}^{(1)}(v) \mathcal{A}^{(1)}(u)+\frac{2 v+1}{2(u-v)(v+1)} \mathcal{B}^{(1)}(u) \mathcal{A}^{(1)}(v)-\frac{1}{u+v+2} \mathcal{B}^{(1)}(u) \tilde{\mathcal{D}}^{(1)}(v), \\
\tilde{\mathcal{D}}^{(1)}(u) \mathcal{B}^{(1)}(v)= & \frac{(u-v+1)(u+v+3)}{(u+v+2)(u-v)} \mathcal{B}^{(1)}(v) \tilde{\mathcal{D}}^{(1)}(u)-\frac{2 u+3}{2(u-v)(u+1)} \mathcal{B}^{(1)}(u) \tilde{\mathcal{D}}^{(1)}(v) \\
& +\frac{(2 u+3)(2 v+1)}{4(u+1)(v+1)(u+v+2)} \mathcal{B}^{(1)}(u) \mathcal{A}^{(1)}(v) .
\end{aligned}
$$

We choose as the nested vacuum state the product state

$$
\left|\Phi^{(1)}\right\rangle=\prod_{l=1}^{M}\left|0^{(1)}\right\rangle_{l}|0\rangle_{\tau}
$$

where $\left|0^{(1)}\right\rangle_{l}=(1,0)^{t_{l}}$ and $|0\rangle_{\tau}=(1,0)^{t_{\tau}}$ are the spin states of the $l$ th site and of the impurity, respectively. Direct calculations imply

$$
\begin{aligned}
\mathcal{A}^{(1)}(u)\left|\Phi^{(1)}\right\rangle & =\frac{2 u(u+1)(u+2)}{2 u+1} a_{0}^{(1)}(u)\left|\Phi^{(1)}\right\rangle, \\
\mathcal{D}^{(1)}(u)\left|\Phi^{(1)}\right\rangle & =\left[u^{2} b_{0}^{(1)}(u)+\frac{u(u+2)}{2 u+1} a_{0}^{(1)}\right]\left|\Phi^{(1)}\right\rangle, \\
\mathcal{C}^{(1)}(u)\left|\Phi^{(1)}\right\rangle & =0, \quad \mathcal{B}^{(1)}(u)\left|\Phi^{(1)}\right\rangle \neq 0,
\end{aligned}
$$

where

$$
a_{0}^{(1)}(u)=\prod_{l=1}^{M}\left(u+u_{l}+2\right)\left(u-u_{l}+1\right), \quad b_{0}^{(1)}(u)=a_{0}^{(1)}(u-1) .
$$

Again, the operator $\mathcal{C}^{(1)}(u)$ can be regraded as the annihilation operator and the operator $\mathcal{B}^{(1)}(u)$ acting on the nested vacuum state, (40), will generate other states. 
Assume that the eigenstate of the nested transfer matrix $t^{(1)}\left(u,\left\{u_{l}\right\}\right)$ is

$$
|F\rangle=\mathcal{B}^{(1)}\left(\mu_{1}\right) \ldots \mathcal{B}^{(1)}\left(\mu_{L}\right)\left|\Phi^{(1)}\right\rangle,
$$

where $\left\{\mu_{k} \mid k=1, \ldots, L\right\}$ are the nested Bethe roots. The nested transfer matrix, (36), acting on the assumed state, (42), gives

$$
\begin{aligned}
t^{(1)}\left(u,\left\{u_{l}\right\}\right)|F\rangle= & \left\{\frac{u(u+2)(2 u+3)}{2 u+1} \prod_{k=1}^{L} \frac{\left(u-\mu_{k}-1\right)\left(u+\mu_{k}+1\right)}{\left(u-\mu_{k}\right)\left(u+\mu_{k}+2\right)} a_{0}^{(1)}(u)+u^{2} \prod_{k=1}^{L} \frac{\left(u+\mu_{k}+3\right)\left(u-\mu_{k}+1\right)}{\left(u-\mu_{k}\right)\left(u+\mu_{k}+2\right)} b_{0}^{(1)}(u)\right\}|F\rangle \\
& +\sum_{k=1}^{L} \Lambda_{k}^{(1)}(u) \mathcal{B}^{(1)}\left(\mu_{1}\right) \ldots \mathcal{B}^{(1)}\left(\mu_{k-1}\right) \mathcal{B}^{(1)}(u) \mathcal{B}^{(1)}\left(\mu_{k+1}\right) \ldots \mathcal{B}^{(1)}\left(\mu_{L}\right)\left|\Phi^{(1)}\right\rangle,
\end{aligned}
$$

where $\Lambda_{k}^{(1)}(u)$ denotes the unwanted term with the form

$$
\begin{aligned}
\Lambda_{k}^{(1)}(u)= & \frac{(2 u+3) \mu_{k}}{\left(u-\mu_{k}\right)\left(u+\mu_{k}+2\right)}\left[\left(\mu_{k}+2\right) \prod_{m \neq k}^{L} \frac{\left(\mu_{k}+\mu_{m}+1\right)\left(\mu_{k}-\mu_{m}-1\right)}{\left(\mu_{k}-\mu_{m}\right)\left(\mu_{k}+\mu_{m}+2\right)}\right. \\
& \left.\times a_{0}^{(1)}\left(\mu_{k}\right)-\mu_{k} \prod_{m \neq k}^{L} \frac{\left(\mu_{k}-\mu_{m}+1\right)\left(\mu_{k}+\mu_{m}+3\right)}{\left(\mu_{k}-\mu_{m}\right)\left(\mu_{k}+\mu_{m}+2\right)} b_{0}^{(1)}\left(\mu_{k}\right)\right] .
\end{aligned}
$$

From Eq. (43), we know that if all the unwanted terms are 0, the assumed state, (42), is indeed the eigenstate of the nested transfer matrix $t^{(1)}\left(u,\left\{\bar{u}_{l}\right\}\right)$. Putting $\Lambda_{k}^{(1)}(u)=0$, we obtain the second set of Bethe ansatz equations

$$
\frac{\left(\bar{\mu}_{k}+i\right)\left(\bar{\mu}_{k}+\frac{1}{2} i\right)}{\left(\bar{\mu}_{k}-i\right)\left(\bar{\mu}_{k}-\frac{1}{2} i\right)} \prod_{m=1}^{L} \frac{\left(\bar{\mu}_{k}-\bar{\mu}_{m}-i\right)\left(\bar{\mu}_{k}+\bar{\mu}_{m}-i\right)}{\left(\bar{\mu}_{k}-\bar{\mu}_{m}+i\right)\left(\bar{\mu}_{k}+\bar{\mu}_{m}+i\right)}=-\prod_{j=1}^{M} \frac{\left(\bar{\mu}_{k}+\bar{u}_{j}-\frac{1}{2} i\right)\left(\bar{\mu}_{k}-\bar{u}_{j}-\frac{1}{2} i\right)}{\left(\bar{\mu}_{k}+\bar{u}_{j}+\frac{1}{2} i\right)\left(\bar{\mu}_{k}-\bar{u}_{j}+\frac{1}{2} i\right)}, \quad k=1,2, \ldots, L,
$$

where we have used the notation $u_{j}=i \bar{u}_{j}-\frac{1}{2}, \mu_{k}=i \bar{\mu}_{k}-1$ and $i$ is the imaginary unit. The corresponding eigenvalue reads

$$
\begin{aligned}
\Lambda^{(1)}\left(u,\left\{\bar{u}_{l}\right\}\right)= & \frac{u(u+2)(2 u+3)}{2 u+1} \prod_{j=1}^{M}\left(u+\bar{u}_{j} i+\frac{3}{2}\right)\left(u-\bar{u}_{j} i+\frac{1}{2}\right) \prod_{k=1}^{L} \frac{\left(u+\bar{\mu}_{k} i\right)\left(u-\bar{\mu}_{k} i-2\right)}{\left(u-\bar{\mu}_{k} i-1\right)\left(u+\bar{\mu}_{k} i+1\right)} \\
& +u^{2} \prod_{j=1}^{M}\left(u+\bar{u}_{j} i+\frac{1}{2}\right)\left(u-\bar{u}_{j} i-\frac{1}{2}\right) \prod_{k=1}^{L} \frac{\left(u-\bar{\mu}_{k} i\right)\left(u+\bar{\mu}_{k} i+2\right)}{\left(u-\bar{\mu}_{k} i-1\right)\left(u+\bar{\mu}_{k} i+1\right)} .
\end{aligned}
$$

Substituting Eq. (46) into Eq. (32), we obtain the explicit form of the first set of Bethe ansatz equations

$$
\frac{\bar{u}_{j}-\frac{3}{2} i}{\bar{u}_{j}+\frac{3}{2} i}\left(\frac{\bar{u}_{j}+\frac{1}{2} i}{\bar{u}_{j}-\frac{1}{2} i}\right)^{2 N+1} \prod_{l=1}^{M} \frac{\left(\bar{u}_{j}-\bar{u}_{l}-i\right)\left(\bar{u}_{j}+\bar{u}_{l}-i\right)}{\left(\bar{u}_{j}-\bar{u}_{l}+i\right)\left(\bar{u}_{j}+\bar{u}_{l}+i\right)}=\prod_{k=1}^{L} \frac{\left(\bar{u}_{j}-\bar{\mu}_{k}-\frac{1}{2} i\right)\left(\bar{u}_{j}+\bar{\mu}_{k}-\frac{1}{2} i\right)}{\left(\bar{u}_{j}-\bar{\mu}_{k}+\frac{1}{2} i\right)\left(\bar{u}_{j}+\bar{\mu}_{k}+\frac{1}{2} i\right)}, \quad j=1,2, \ldots, M .
$$

Substituting Eq. (46) into Eq. (33), we obtain the eigenvalues $\Lambda(u)$ of the transfer matrix $t(u)$

$$
\begin{aligned}
\Lambda(u)= & \frac{(2 u+3)\left(1-u^{2}\right)}{2 u+1} \prod_{j=1}^{M} \frac{\left(u-\bar{u}_{j} i-\frac{3}{2}\right)\left(u+\bar{u}_{j} i-\frac{1}{2}\right)}{\left(u+\bar{u}_{j} i+\frac{1}{2}\right)\left(u-\bar{u}_{j} i-\frac{1}{2}\right)}+u^{2} b_{0}(u) \prod_{k=1}^{L} \frac{\left(u+\bar{\mu}_{k} i+2\right)\left(u-\bar{\mu}_{k} i\right)}{\left(u-\bar{\mu}_{k} i-1\right)\left(u+\bar{\mu}_{k} i+1\right)} \\
& +\frac{u(u+2)(2 u+3)}{2 u+1} b_{0}(u) \prod_{j=1}^{M} \frac{\left(u+\bar{u}_{j} i+\frac{3}{2}\right)\left(u-\bar{u}_{j} i+\frac{1}{2}\right)}{\left(u+\bar{u}_{j} i+\frac{1}{2}\right)\left(u-\bar{u}_{j} i-\frac{1}{2}\right)} \prod_{k=1}^{L} \frac{\left(u-\bar{\mu}_{k} i-2\right)\left(u+\bar{\mu}_{k} i\right)}{\left(u-\bar{\mu}_{k} i-1\right)\left(u+\bar{\mu}_{k} i+1\right)},
\end{aligned}
$$

where the Bethe roots $\left\{\bar{u}_{j}\right\}$ and $\left\{\bar{\mu}_{k}\right\}$ should satisfy the Bethe ansatz equations, (45) and (47). The eigenenergy of Hamiltonian (1) is

$$
E=\left.\frac{1}{6} \frac{d \Lambda(u)}{d u}\right|_{u=0}+2 N-\frac{11}{6}=-\sum_{j=1}^{M} \frac{1}{\bar{u}_{j}^{2}+\frac{1}{4}}+2 N-\frac{5}{2}
$$

\section{CONCLUSION}

In this paper, we construct the operator solution of the reflection equation. Based on it, we propose an integrable quantum spin chain with a magnetic impurity. By using the nested algebraic Bethe ansatz, we obtain the exact solution of the system.

The scheme given in this paper can be generalized. For example, if we start from the trigonometric or elliptic $R$ matrix, we can construct an integrable anisotropic model with 
an impurity. Then the anisotropic effects of the magnetic impurity can be studied. Meanwhile, the bulk of model (1) has $\mathrm{SU}(3)$ symmetry. If we start from the $R$ matrix of the SU(2)-invariant high-spin Heisenberg model, we can put the integrable impurity into the $\mathrm{SU}(2)$-invariant high-spin Heisenberg model. We also note that if both sides of the open systems have impurities, the corresponding exact solution can also be studied similarly.

Another interesting issue is that the reflection equation may have other forms of operator solutions. One particular case is that the reflection matrix may have nondiagonal elements, which will change the spin states of quasiparticles after reflection by the boundaries. Then the U(1) symmetry of the system is broken and the nested algebraic Bethe ansatz does not work because the reference state, (24), is no longer valid. In this case, we expect that the off-diagonal Bethe ansatz [51,52] could be applied.

\section{ACKNOWLEDGMENTS}

We would like to thank Prof. Y. Wang for his valuable discussions and continuous encouragement. The financial support from the National Program for Basic Research of MOST (Grants No. 2016YFA0300600 and No. 2016YFA0302104), the National Natural Science Foundation of China (Grants No. 12074410, No. 12047502, No. 11934015, No. 11975183, No. 11774397 , No. 11775178 , and No. 11775177), the Major Basic Research Program of Natural Science of Shaanxi Province (Grants No. 2017KCT12 and No. 2017ZDJC-32), the Strategic Priority Research Program of the Chinese Academy of Sciences (Grant No. XDB33000000), and the fellowship of China Postdoctoral Science Foundation (Grant No. 2020M680724) is gratefully acknowledged.
[1] T. Giamarchi, Quantum Physics in One Dimension (Oxford University Press, Oxford, UK, 2003).

[2] S. R. White, Density-matrix algorithms for quantum renormalization groups, Phys. Rev. B 48, 10345 (1993).

[3] U. Schollwöck, The density-matrix renormalization group, Rev. Mod. Phys. 77, 259 (2005)

[4] C. N. Yang, Some Exact Results for the Many-Body Problem in One Dimension with Repulsive Delta-Function Interaction, Phys. Rev. Lett. 19, 1312 (1967); C. N. Yang, S-matrix for the one-dimensional $\mathrm{N}$-body problem with repulsive or attractive $\delta$-function interaction, Phys. Rev. 168, 1920 (1968).

[5] E. H. Lieb and F. Y. Wu, Absence of Mott Transition in an Exact Solution of the Short-Range, One-Band Model in One Dimension, Phys. Rev. Lett. 20, 1445 (1968).

[6] A. Föerster and M. Karowski, Algebraic properties of the Bethe ansatz for an $\operatorname{spl}(2,1)$ supersymmetric t-J model, Nucl. Phys. B 396, 611 (1993).

[7] L. A. Takhtadzhan and L. D. Faddeev, The quantum method of the inverse problem and the Heisenberg XYZ model, Russ. Math. Surv. 34, 11 (1979).

[8] H. A. Bethe, Zur Theorie der Metalle I. Eigenwerte und Eigenfunktionen der linearen Atomkette, Z. Phys. 71, 205 (1931).

[9] M. Gaudin, The Bethe Wavefunction (Cambridge University Press, Cambridge, UK, 2014).

[10] E. K. Sklyanin and L. D. Faddeev, Quantum mechanical approach to completely integrable field theory models, Sov. Phys. Dokl. 23, 902 (1978).

[11] L. D. Faddeev, Quantum completely integrable models in field theory, Sov. Sci. Rev. Math. C 1, 107 (1980).

[12] P. P. Kulish and E. K. Sklyannin, Quantum spectral transform method. Recent developments, Lect. Notes Phys. 151, 61 (1982).

[13] E. K. Sklyanin, Quantum version of the method of inverse scattering problem, J. Sov. Math. 19, 1546 (1982).

[14] R. J. Baxter, Exactly Solved Models in Statistical Mechanics (Academic Press, London, 1982).

[15] B. Sutherland, Further Results for the Many-Body Problem in One Dimension, Phys. Rev. Lett. 20, 98 (1968).
[16] B. Sutherland, Model for a multicomponent quantum system, Phys. Rev. B 12, 3795 (1975).

[17] V. E. Korepin, N. M. Boliubov, and A. G. Izergin, Quantum Inverse Scattering Method and Correlation Functions (Cambridge University Press, Cambridge, UK, 1993).

[18] N. Andrei, Diagonalization of the Kondo Hamiltonian, Phys. Rev. Lett. 45, 379 (1980).

[19] P. B. Wiegmann, Exact solution of s-d exchange model at $\mathrm{T}=$ 0, Sov. Phys. JETP Lett. 31, 364 (1980).

[20] V. A. Fateev and P. B. Wiegmann, The exact solution of the s-d exchange model with arbitrary impurity spin S (Kondo problem), Phys. Lett. A 81, 179 (1981).

[21] H. M. Babujian, Exact solution of the one-dimensional isotropic Heisenberg chain with arbitrary spins S, Phys. Lett. A 90, 479 (1982).

[22] P. B. Wiegmann and A. M. Tsvelick, Exact solution of the Anderson model: I, J. Phys. C: Solid State Phys. 16, 2281 (1983).

[23] A. M. Tsvelik and P. B. Wiegmann, Exact results in the theory of magnetic alloys, Adv. Phys. 32, 453 (1983).

[24] N. Andrei and H. Johannesson, Heisenberg chain with impurities (an integrable model), Phys. Lett. A 100, 108 (1984).

[25] N. Andrei and C. Destri, Solution of the Multichannel Kondo Problem, Phys. Rev. Lett. 52, 364 (1984).

[26] A. M. Tsvelick and P. B. Wiegmann, Exact Solution of the Multichannel Kondo Problem, Scaling, and Integrability, J. Stat. Phys. 38, 125 (1985).

[27] Y. Wang and J. Voit, An Exactly Solvable Kondo Problem for Interacting One-Dimensional Fermions, Phys. Rev. Lett. 77, 4934 (1996).

[28] Y. Wang, J. Dai, Z. Hu, and F.-C. Pu, Exact Results for a Kondo Problem in a One-Dimensional t-J Model, Phys. Rev. Lett. 79, 1901 (1997).

[29] Y. Wang, Exact solution of the open Heisenberg chain with two impurities, Phys. Rev. B 56, 14045 (1997).

[30] A. Jerez, N. Andrei, and G. Zaránd, Solution of the multichannel Coqblin-Schrieffer impurity model and application to multilevel systems, Phys. Rev. B 58, 3814 (1998). 
[31] C. Rylands and N. Andrei, Quantum dot at a Luttinger liquid edge, Phys. Rev. B 96, 115424 (2017); Quantum dot in interacting environments, 97, 155426 (2018).

[32] P. R. Pasnoori, C. Rylands, and N. Andrei, Kondo impurity at the edge of a superconducting wire, Phys. Rev. Res. 2, 013006 (2020).

[33] I. Cherednik, Factorizing particles on a half line and root systems, Theor. Math. Phys. 61, 977 (1984).

[34] I. Cherednik, Some finite-dimensional representations of generalized Sklyanin algebras, Funct. Anal. Appl. 19, 77 (1985).

[35] E. K. Sklyanin, Boundary conditions for integrable quantum systems, J. Phys. A 21, 2375 (1988).

[36] L. Mezincescu and R. I. Nepomechie, Analytical Bethe ansatz for quantum-algebra-invariant spin chains, Nucl. Phys. B 372, 597 (1992).

[37] Y.-K. Zhou, Row transfer matrix functional relations for Baxter's eight-vertex and six-vertex models with open boundaries via more general reflection matrices, Nucl. Phys. B 458, 504 (1996).

[38] A. Lima-Santos and R. Malara, $C_{n}^{(1)}, D_{n}^{(1)}$ and $A_{2 n-1}^{(2)}$ reflection K-matrices, Nucl. Phys. B 675, 661 (2003).

[39] R. Malara and A. Lima-Santos, On $\mathcal{A}_{n-1}^{(1)}, \mathcal{B}_{n}^{(1)}, \mathcal{C}_{n}^{(1)}, \mathcal{D}_{n}^{(1)}, \mathcal{A}_{2 n}^{(2)}$, $\mathcal{A}_{2 n-1}^{(2)}$, and $\mathcal{D}_{n+1}^{(2)}$ reflection K-matrices, J. Stat. Mech. (2006) $\mathrm{P} 09013$.

[40] H.-Q. Zhou, X.-Y. Ge, J. Links, and M. D. Gould, Graded reflection equation algebras and integrable Kondo impurities in the one-dimensional t-J model, Nucl. Phys. B 546, 779 (1999).

[41] F. D. M. Haldane, Continuum dynamics of the 1-D Heisenberg antiferromagnet: Identification with the $\mathrm{O}(3)$ nonlinear sigma model, Phys. Lett. A 93, 464 (1983).
[42] F. D. M. Haldane, Nonlinear Field Theory of Large-Spin Heisenberg Antiferromagnets: Semiclassically Quantized Solitons of the One-Dimensional Easy-Axis Néel State, Phys. Rev. Lett. 50, 1153 (1983).

[43] F. D. M. Haldane, " $\Theta$ physics" and quantum spin chains (abstract), J. Appl. Phys. 57, 3359 (1985).

[44] I. Affleck, T. Kennedy, E. H. Lieb, and H. Tasaki, Rigorous Results on Valence-Bond Ground States in Antiferromagnets, Phys. Rev. Lett. 59, 799 (1987).

[45] I. Affleck, T. Kennedy, E. H. Lieb, and H. Tasaki, Valence bond ground states in isotropic quantum antiferromagnets, Commun. Math. Phys. 115, 477 (1988).

[46] K. Duivenvoorden and T. Quella, Topological phases of spin chains, Phys. Rev. B 87, 125145 (2013).

[47] M. Hagiwara, K. Katsumata, I. Affleck, B. I. Halperin, and J. P. Renard, Observation of $S=1 / 2$ Degrees of Freedom in an $S=1$ Linear-Chain Heisenberg Antiferromagnet, Phys. Rev. Lett. 65, 3181 (1990).

[48] H. Frahm and N. A. Slavnov, New solutions to the reflection equation and the projecting method, J. Phys. A 32, 1547 (1999).

[49] W. Galleas and M. J. Martins, Solution of the SU(N) vertex model with nondiagonal open boundaries, Phys. Lett. A 335, 167 (2005).

[50] H. J. de Vega and A. González-Ruiz, Boundary K-matrices for the XYZ, XXZ and XXX spin chains, J. Phys. A 27, 6129 (1994).

[51] J. Cao, W.-L. Yang, K. Shi, and Y. Wang, Off-Diagonal Bethe Ansatz and Exact Solution of a Topological Spin Ring, Phys. Rev. Lett. 111, 137201 (2013).

[52] Y. Wang, W.-L. Yang, J. Cao, and K. Shi, Off-Diagonal Bethe Ansatz for Exactly Solvable Models (Springer Verlag, Berlin, 2015). 